\title{
Evolutionary Timescales of AO-Produced Speckles at NIR Wavelengths
}

\author{
Sean B. Goebel ${ }^{\mathrm{a}, \mathrm{b}}$, Olivier Guyon ${ }^{\mathrm{b}, \mathrm{c}, \mathrm{d}, \mathrm{e}}$, Donald N.B. Hall ${ }^{\mathrm{a}}$, Nemanja Jovanovic ${ }^{\mathrm{b}, \mathrm{f}}$, and Dani E. \\ Atkinson $^{\text {a }}$ \\ anstitute for Astronomy, University of Hawaii, 640 North A'ohoku Place, Hilo, HI 96720 \\ ${ }^{b}$ Subaru Telescope, National Astronomical Observatory of Japan, 650 North A'ohoku Place, \\ Hilo, HI 96720 \\ 'Steward Observatory, University of Arizona, Tucson, AZ 85721 \\ ${ }^{\mathrm{d} C}$ College of Optical Sciences, University of Arizona, Tucson, AZ 85721 \\ eAstrobiology Center of NINS, 2-21-1, Osawa, Mitaka, Tokyo, 181-8588, Japan \\ ${ }^{\mathrm{f}}$ Department of Physics and Astronomy, Macquarie University, Sydney, NSW 2109, Australia
}

\begin{abstract}
We present measurements of the evolutionary timescales of speckles around adaptive optics-corrected PSFs. We placed a SELEX SAPHIRA HgCdTe detector behind the SCExAO instrument at Subaru Telescope. We analyzed the behavior of speckles at radial distances of 2-8 $\lambda / D$ away from the diffraction-limited PSF in H-band $(\sim 1.6 \mu \mathrm{m})$ images collected at $\sim 1 \mathrm{kHz}$ framerates. Speckles evolve with a variety of timescales, and these have not previously been studied at near-infrared wavelengths. Ultimately we would like to image reflected-light exoplanets, which necessitates a fast speckle control loop. Based on our measurements, we calculate the parameters of an optimized control loop that would enable such observations.
\end{abstract}

Keywords: Extreme adaptive optics, SCExAO, SAPHIRA, speckles, high-contrast imaging

\section{INTRODUCTION}

Adaptive optics-corrected point spread functions (PSFs) consist of a core with an angular size determined by the diffraction limit of the telescope and a surrounding halo of speckles with an angular size matching that of the atmospheric seeing. The speckles are caused by imperfect $\mathrm{AO}$ corrections and diffraction within the optical system of the telescope and instrument. The imperfections in adaptive optics stem from measurement noise (due to detector and photon noise), fitting error (the inability of the system to measure and correct all possible spatial frequencies of aberrations), and insufficient bandwidth (the delay between measurement and correction). ${ }^{1}$ As long as these issues are present, speckles will surround the target being observed. In an extreme adaptive optics system (Strehl 90\%), the typical brightness of a speckle is about $10^{-3}$ times that of the PSF core, and bright speckles can reach the $10^{-2}$ level. Strehl is defined as the ratio of the intensity at the center of an image of an aberrated point source to the intensity of a PSF that has been diffracted by the system's aperture but otherwise has no aberrations. Speckles evolve with a variety of lifetimes; on one extreme, quasi-static speckles are caused by diffraction within the optics or mis-calibration of the $\mathrm{AO}$ system, and at the other extreme, measurement noise creates speckles that evolve on timescales on the order of the $\mathrm{AO}$ update rate. A number of attempts have been made with simulations or visible-wavelength imaging to characterize the lifetimes of speckles, ${ }^{1-3}$ but this hasn't been examined at NIR wavelengths, which is where most extreme-AO science instruments observe.

Speckles are similar in size to potential companions to a target, and they wash out faint features in extended sources. Planetary companions or faint features will be lost in the speckles unless steps are taken to attenuate the speckles. A number of techniques have been developed to achieve this in order to study high-contrast objects. Angular differential imaging ${ }^{4}$ takes advantage of the rotation of the sky relative to an alt-az telescope in order to subtract off fixed-pattern aberrations. Spectral differential imaging ${ }^{5}$ and polarimetric differential imaging ${ }^{6}$

Send correspondence to Sean Goebel, sgoebel@ifa.hawaii.edu 
are two related techniques that utilize color and polarization information, respectively, to separate companions from speckles caused by scattered star light. However, these techniques are all done in post-processing, and they only remove quasi-static speckles. Quickly-changing speckles smear into a smooth halo.

Active speckle control techniques are a way to enable observations of targets for which angular differential imaging and similar post-processing techniques do not provide sufficient contrast improvements. Martinache et al. $(2014)^{7}$ demonstrated a real-time technique to null away speckles in one half of the frame at a time. This technique, which had an on-sky update rate on the order of $\sim 1 \mathrm{~Hz}$, successfully produced a "dark hole" of reduced speckles near the PSF. The effectiveness of a real-time speckle control loop depends largely on its update rate (a faster-updating loop can attenuate shorter-lifetime speckles).

In this paper, we present measurements of the lifetimes of speckles at near-infrared (NIR) wavelengths. We then apply these measurements to calculate the parameters of a theoretical real-time speckle-nulling loop that would produce various levels of raw contrasts. New speckle-reduction techniques will need to be implemented in order to study objects of ever-increasing contrasts, and we provide requirements for future active speckle-nulling control loops.

\section{EXPERIMENTAL SETUP AND DATA REDUCTION}

\subsection{SCExAO and the SAPHIRA Detector}

The Subaru Coronagraphic Extreme Adaptive Optics (SCExAO) instrument ${ }^{8}$ is a project at the $8.2 \mathrm{~m}$ Subaru Telescope atop Mauna Kea. It uses coronagraphic techniques and high-order adaptive optics corrections ${ }^{9}$ to directly image high-contrast targets such as extrasolar planets separated from their host stars by angles near the diffraction limit of the telescope. SCExAO makes use of light from $600 \mathrm{~nm}$ to $1.8 \mu \mathrm{m}$.

SCExAO is placed behind Subaru's AO188 (188-element adaptive optics) system, which provides a preliminary AO correction. AO188 Strehl is typically around $30-40 \%$ in $\mathrm{H}$ band under median seeing conditions, ${ }^{10}$ and SCExAO increases this to $~ 90 \%$. SCExAO's AO corrections are computed using several GPUs running in parallel, and the correction is applied to a 2000-element Boston MicroMachines MEMS deformable mirror at an update rate of $3.5 \mathrm{kHz}$.

After receiving light from AO188 and providing additional corrections, SCExAO splits it into optical and infrared bands so that both optical and infrared instruments can be run simultaneously. On the infrared side, the normal science instrument is HiCIAO (High-Contrast Coronographic Imager for Adaptive Optics), ${ }^{11}$ which is a Subaru facility instrument utilizing an H2RG detector. HiCIAO can be removed and replaced with SAPHIRA, which is the detector which produced the data we report on here. On the visible side, SCExAO sends AOcorrected beams to VAMPIRES (Visible Aperture Masking Polarimetric Interferometer for Resolving Exoplanetary Signatures) ${ }^{12}$ and FIRST (Fibered Imager foR a Single Telescope), ${ }^{13}$ which are aperture-masking interferometers.

SAPHIRA (Selex Advanced Photodiode array for High speed InfraRed Arrays) ${ }^{14}$ is an infrared avalanche photodiode detector manufactured by Leonardo (which until recently was called Selex). It has $320 \times 256 \times 24 \mu \mathrm{m}$ metal organic vapor phase epitaxy (MOVPE) mercury cadmium telluride ( $\mathrm{HgCdTe})$ pixels and 32 readout channels. The avalanche gain can be set as high as 600 , which enables the potential for sub-electron read noise and photon counting operation. The avalanche gain (which has units of outputted electrons/detected photon) is different from the charge gain (which has units of electrons/ADU or microvolts/ADU). The avalanche gain is the multiplication factor of the signal from a photoelectron and occurs within the multiplication layer. The charge gain is the conversion from the electrons collected at the integrating node to ADU. The charge gain is currently fixed for the SAPHIRA at a value of $\sim 2.9$ e-/ADU, whereas the avalanche gain can be adjusted by varying the bias across the photodiode. At low avalanche gains, SAPHIRA has more dynamic range, fewer hot pixels, and better uniformity. The read noise (in electrons) is approximately constant at the various avalanche gains, so at high avalanche gains it decreases relative to the signal from the photons. At significantly high gains, the signal of an individual photon is greater than the read noise, offering the potential for photon counting. The dark current is $\ll 1 \mathrm{e}^{-} / \mathrm{s}$ at low avalanche gains.

The SAPHIRA detector is sensitive to 0.8-2.5 $\mu \mathrm{m}$ light, and our observations were collected at $\mathrm{H}$ band $(1.6 \mu \mathrm{m})$. The maximum readout rate of SAPHIRA exceeds 10 megapixels/second for each of the 32 readouts, which 
corresponds to a full-frame readout rate of around $4 \mathrm{kHz}$. We read out the detector using a Leach controller, ${ }^{15}$ which permitted a full-frame readout rate of approximately $100 \mathrm{~Hz}$. Subarrays within SAPHIRA can be selected in order to increase the frame rate, and we read out about $1 / 13$ of the detector in order to achieve $\sim 1.4 \mathrm{kHz}$ readout rates. In the future we expect to upgrade the readout electronics to the "Pizza Box" currently under development to achieve faster readout rates.

\subsection{Observations}

We collected speckle data on the night of March 18, 2016 during observations of alf Boo (also known as Arcturus), which has a brightness of $H=-2.81$. The weather was clear, median $0.5 \mu \mathrm{m}$ seeing was 0.7 ", and SCExAO was producing a tip-tilt-corrected Strehl of approximately $50 \%$ at $\mathrm{H}$ band. This somewhat suboptimal Strehl was caused by imperfect instrument tuning; SCExAO is still in development and undergoes frequent software and hardware changes. The pixel scale of SAPHIRA was $10.7 \mathrm{mas} /$ pixel. Given that $\lambda / D=40.7 \mathrm{mas}$ for the $8.2 \mathrm{~m}$ diameter of Subaru at $\mathrm{H}$ band, the detector's pixels sample the image at approximately double the Nyquist frequency. We analyzed two 10,000-image cubes with AO188 + SCExAO's 2000-element AO system operating, 10,000 images with only AO188 running, and 10,000 images with no adaptive optics corrections. These were collected near in time to each other, so the atmospheric seeing conditions did not change significantly. We read a 96x64 pixel subwindow, enabling a $1380 \mathrm{~Hz}$ framerate.

\subsection{Data Reduction}

SAPHIRA operated in an "up-the-ramp" readout mode, meaning that it was read out multiple times between each reset. We set the detector to read six times between each reset, which provided an optimal tradeoff between duty cycle (the first frame after reset had excess noise and was discarded) and dynamic range (the $\sim 40,000 \mathrm{ADU}$ dynamic range needed to be split across those six frames). Due to the high framerate and an over-taxed computer, a small fraction $(0.1 \%)$ of the frames were dropped at random.

We wrote Python code to identify where in the data cubes the detector resets occurred. Because the detector operated in up-the-ramp readout mode, subtracting adjacent images removes common noise and leaves the flux that accumulated between the two reads. The first frame following each reset was discarded due to its excess noise, and each remaining image was subtracted from the one collected immediately after it. In this way, a sixframe reset frequency yields five correlated double slope (CDS) pairs, and four of these are useful for analysis. The duty cycle therefor was $67 \%$. During observations, the timestamp of each recorded frame was saved; we referenced these to detect when the computer had dropped frames. In order to maintain regular time sampling for later analysis, we recorded where data was missing due to discarded and dropped frames.

Next, we performed an optimized centroid calculation ${ }^{16}$ on the PSF in each image and shifted the images to remove tip and tilt. We are interested in the evolution of speckles over time, and tip and tilt merely translate the speckles around, would dominate the speckle lifetime results, and are highly dependent on the tuning of the AO system, so we removed tip and tilt. Next, we formed annular masks with radii $2 \lambda / D<r<8 \lambda / D$. Sample individual and coadded images are shown in Figure 1.

\section{ANALYSIS}

\subsection{Speckle Lifetimes}

In each image, we selected the $10 \%$ and $80 \%$ dimmest pixels within the $2 \lambda / D<r<8 \lambda / D$ annular mask, thereby selecting the areas not occupied with speckles. We are curious how long the dark areas of the image stay dark, since this is what matters for observing dim targets such as extrasolar planets. We chose the $10 \%$ and $80 \%$ threshold levels to compare how brighter areas change relative to dimmer areas.

Next, we subtracted those pixels from the same pixels at later times (thereby forming difference images) and computed the spatial standard deviation of pixel brightnesses. This quantity was computed for times ranging from one frame difference $(0.72 \mathrm{~ms})$ to 260 frames $(\sim 0.2 \mathrm{~s})$. The standard deviations were divided by the mean brightness of the PSF core, thereby converting them to relative contrasts. This also removes the scaling of the detector gain. This was repeated for every frame in the 10,000-frame cube, and the results were averaged in order to reduce noise in the measurements. We then fit second-order (quadratic) polynomials to the second 

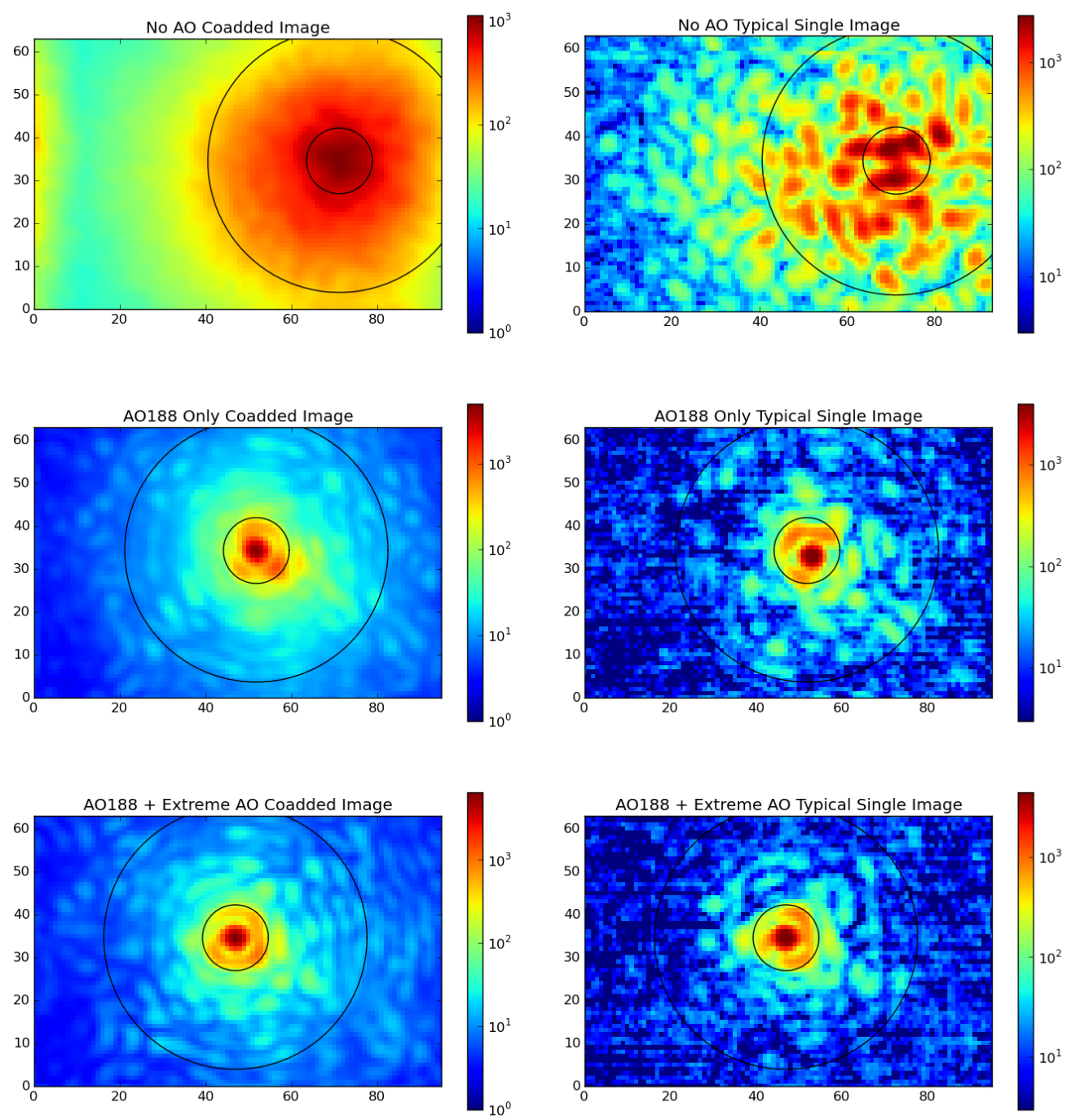

Figure 1. Shown here are single and coadded SAPHIRA images for each of the three AO cases. All images have been logarithmically scaled in order to reveal the faint speckle halos. The black circles are located at $r=2 \lambda / D$ and $r=8 \lambda / D$ and indicate the region over which the speckle calculations were performed. The coadded 10,000 frames images are shown in the left column, and typical (50th percentile Strehl) single frames are shown on the right. From top to bottom are images showing the results of no AO, AO188 corrections only, and the corrections of AO188 + SCExAO. The slight brightening on the left side of the no AO coadded image is an artifact of the tip/tilt correction in post-processing; only the region inside the annulus was analyzed, so this artifact did not affect the results. More light was directed to SAPHIRA for the no AO images, which is why its peak flux is similar to that of the other images. It is worth noting that the AO188 + Extreme AO images have better-defined Airy rings and a smaller speckle halo than the AO188-only images.

through twentieth data points. The first data point (corresponding to adjacent CDS frames being subtracted) was excluded from the fit because the noise for that case is correlated, ${ }^{*}$ and that has the effect of increasing the point's

* Consider the images immediately following a detector reset. Frame 1 is discarded due to excess noise. Frame 2 is subtracted from frame 3 to form the first CDS pair, frame 3 is subtracted from frame 4 to form a second CDS pair, and frame 4 is subtracted from frame 5 to form a third pair. The one time step difference image (the minimum time sample we are able to probe) is the difference between two adjacent CDS pairs, i.e. $\left(f_{4}-f_{3}\right)-\left(f_{3}-f_{2}\right)=f_{4}-2 f_{3}+f_{2}$. If the standard deviation of the read noise at one pixel of one read is $\sigma$, then in this example the total noise of the difference image is $\sigma_{\text {total }}^{2}=\sigma^{2}+(2 \sigma)^{2}+\sigma^{2}=6 \sigma^{2}$. On the other hand, the difference image corresponding to two time steps would 
standard deviation. We fit the data points with a quadratic polynomial because the intensity we measure is the square of complex amplitude, and complex amplitude changes linearly with time for short timescales (timescales on the order of the bandwidth of the AO system), so the measured intensity should change quadratically with time. Lastly, we subtracted off the y-intercepts of the fitted lines. The y-intercepts correspond to the read and photon noise, and therefore are not a parameter we consider when deriving bandwidth for speckle nulling loops. The resulting speckle standard deviation vs. time plots are shown in Figures 2, 3, and 4.

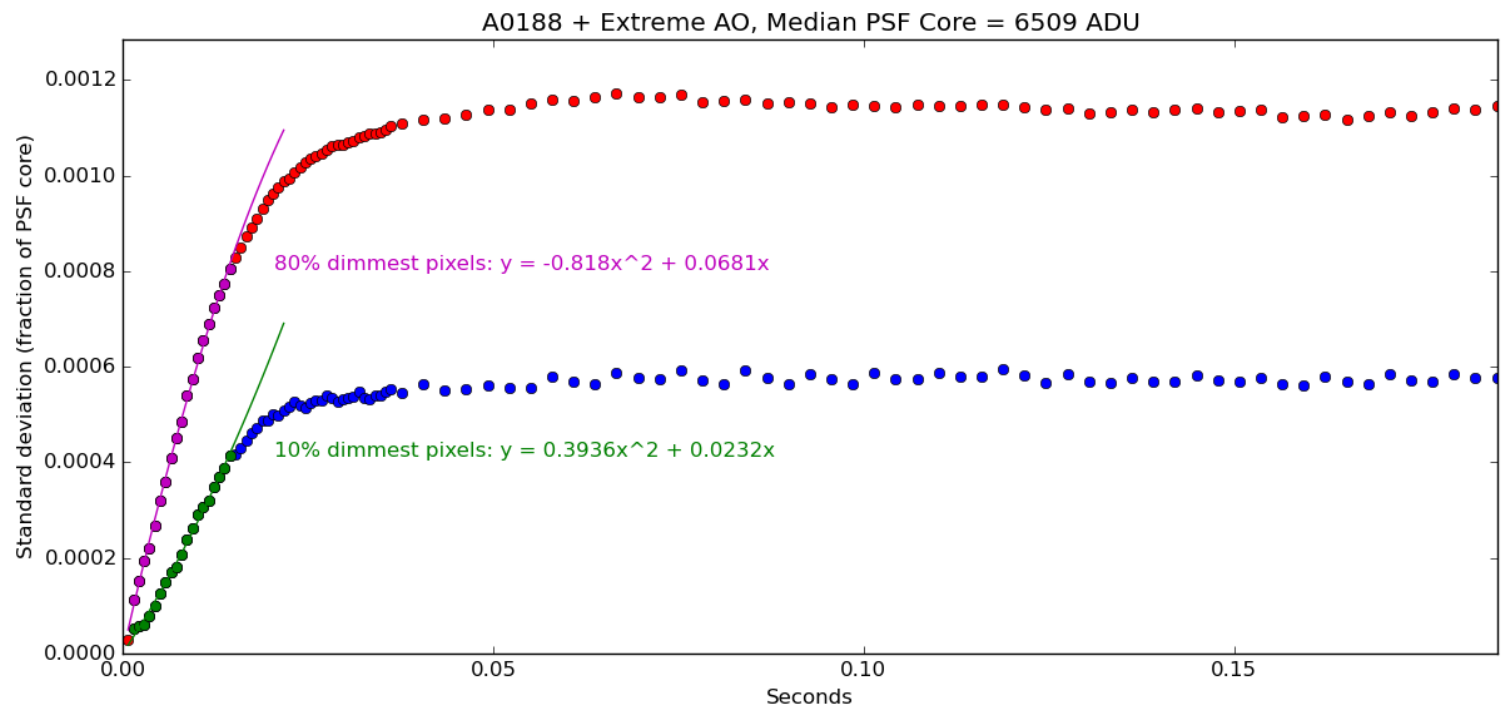

Figure 2. Plotted here are the intensity standard deviations (as a fraction of the PSF core brightness) of differences of two images vs. the time separating those two images. The standard deviations were calculated within a $2 \lambda / D<r<8 \lambda / D$ annulus of the PSF core for the $10 \%$ and $80 \%$ dimmest pixels. The points to which the second-order polynomial was fit are indicated in magenta and green. The standard deviation was calculated at coarser time sampling beginning around 0.03s in order to speed the computation. At long timescales $(t \gtrsim 0.03 \mathrm{~s})$, the speckles have evolved and bear little resemblance to their previous form, so the standard deviation approaches an asymptotic value. Note that the dimmer speckles evolve quadratically with time-this is discussed in Section 3.1.

\subsection{Interpretation for Future Speckle Nulling Loops}

We can solve the equations for the fitted lines in Figure 2 to derive what bandwidth would be needed for a speckle nulling loop to achieve certain raw contrasts. Setting $y=10^{-4}$ (i.e. keeping the speckles stable to a $10^{-4}$ raw contrast) and solving the quadratic equation for $\mathrm{x}$ yields a speckle nulling loop bandwidth of $\sim 700 \mathrm{~Hz}$ for the $80 \%$ dimmest pixels and $\sim 250 \mathrm{~Hz}$ for the $10 \%$ dimmest pixels. In closed loop speckle nulling operation, the linear term is zeroed out with each iteration, so contrast increases with the square of the loop speed. Therefore $10^{-6}$ raw contrast could be achieved for the $10 \%$ dimmest speckles with a loop bandwidth of $\sim 2.5 \mathrm{kHz}$.

It is worth noting that these results are dependent on many variables. Different AO update rates, deformable mirror actuator counts, gains, wind speeds, and other systematic and environmental conditions can all shift these numbers. If the Strehl ratio was higher than the $\sim 50 \%$ it was in the data used for these measurements, the speckles would have been dimmer. A greater fraction of the image would behave as our $10 \%$ dimmest pixels did, so the speckle loop bandwidth could be lower. The speckle nulling loop would crush the brightest speckles, and what remains will be dimmer speckles which require a lower bandwidth loop to null. Therefore the $10 \%$

be $\left(f_{5}-f_{4}\right)-\left(f_{3}-f_{2}\right)$, and the total noise for this case would be $\sigma_{\text {total }}^{2}=\sigma^{2}+\sigma^{2}+\sigma^{2}+\sigma^{2}=4 \sigma^{2}$. All larger time steps would also have a total noise of $4 \sigma^{2}$. Therefore, the standard deviation of the noise of the first point would be a factor of $\sqrt{6 / 4}=\sqrt{3 / 2}$ higher than that of all later points. We therefore decreased the first data point in each of the plots by this factor. However, this correction assumes that all noise is read noise and there is no photon noise, which is close but not entirely correct. Therefore we excluded the first data point from the quadratic fit. 


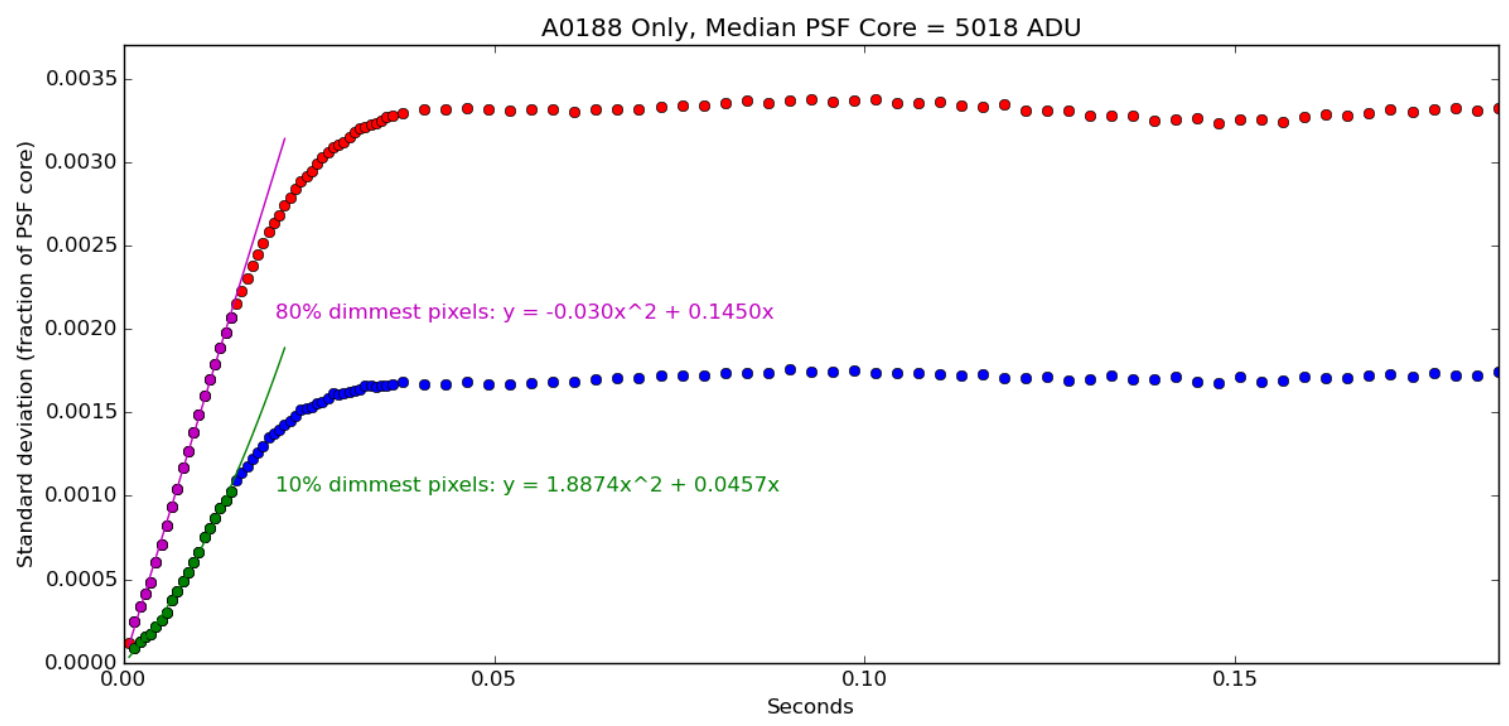

Figure 3. The quantities plotted are the same as in Figure 2, but this time only AO188 is providing corrections. Without the suppressing effects of the high-order SCExAO corrections, the speckles change brightness much more quickly. The mean PSF core was 1500ADU dimmer than the Extreme AO case, indicating that SCExAO was boosting the Strehl by a factor of $\sim 1.3$.

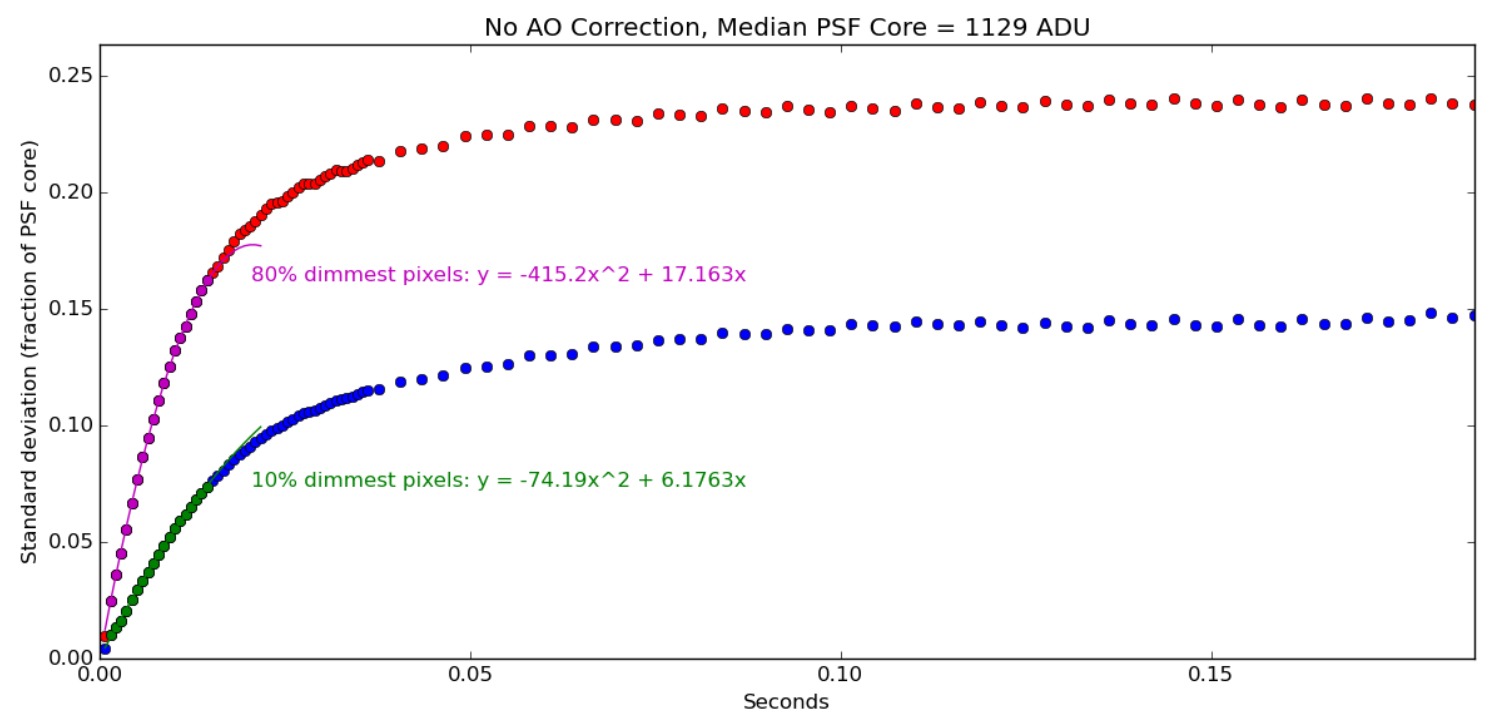

Figure 4. The quantities plotted are the same as in Figure 2, but this time there are no AO corrections. The speckles change at a much greater rate when the PSF is seeing-limited.

dimmest speckles are more representative of closed-loop requirements than the $80 \%$ dimmest pixels. As one nulls increasingly dim speckles, the quadratic term increasingly dominates over the linear term, so the bandwidth requirement approaches an asymptotic value - not zero.

Although the several hundred $\mathrm{Hz}$ bandwidth calculated here is faster than the frame rates of NIR detectors currently deployed and in use, it is not insurmountable. The SAPHIRA detector, which was used with a Leach Controller readout electronics for the data presented here, is currently being tested with an upgraded "Pizza Box" readout that is in development at the University of Hawaii Institute for Astronomy. The Pizza Box will read SAPHIRA in full-frame mode at $\sim 800 \mathrm{~Hz}$, and reading a subwindow of the detector will enable proportionally 
higher readout rates. Also, the Pizza Box controller is not limited to up-the-ramp readout modes. The Pizza Box will operate SAPHIRA in a read-reset-read mode that is much better suited for wavefront sensing applications than the Leach Controller's up-the-ramp mode.

Microwave kinetic inductance detectors (MKIDs) are another promising technology for running rapid speckle nulling loops. They are crygenic detectors capable of detecting individual photons and resolving their energy, and have no dark current or read noise. ${ }^{17}$ Spectral resolution is $R=\lambda / \Delta \lambda \sim 10$ at $4000 \AA$, and temporal resolution is on the order of microseconds. MKIDs are well-suited for low-resolution imaging spectroscopy and exoplanet studies, ${ }^{18}$ and a 20,000-pixel MKIDs array will be delivered to SCExAO in early 2017.

However, even with the fastest detectors and readout electronics, one cannot avoid the fundamental limit on loop speeds imposed by the photon limit. With too short of an integration time (or, equivalently, too dim of a target being observed), simply not enough photons will be detected to identify and null the speckle. A photon-counting detector such as SAPHIRA or MKIDs could reasonably identify a speckle with as few as 10 photons, so this is the speckle detection limit we used. We calculated the contrast of speckles that could be nulled as a function of frame rate and magnitude of the star. Qualitatively, if one wants to detect a speckle at a high contrast, in order to accumulate sufficient photons, the loop must be run slowly (enabling a greater integration time) or the target being observed must be brighter. The quantitative form of this is

$$
S=\frac{C T A F d \lambda}{2.51^{H} N}
$$

where $S$ is the bandwidth of the loop in $\mathrm{Hz}, C$ is the contrast of a speckle relative to the PSF core, $T$ is the throughput of the telescope and instrument, $A$ is the collecting area of the telescope, $F$ is the flux of 0 th magnitude star in units of photons $m^{-2} s^{-1} \mu m^{-1}, d \lambda$ is the bandpass of the filter, $H$ is the magnitude of the target in that filter, and $N$ is the number of photons necessary to detect a speckle.

We then combined this equation with the equation we measured in Figure 2 (setting $y=C$ and $x=1 / S$ ) and solved for achievable contrast as a function of stellar magnitude. The result is shown in Figure 5 .

\section{CONCLUSIONS}

By looking at the temporal evolution of speckles in SCExAO data, we found that a speckle nulling loop with a bandwidth on the order of a few hundred $\mathrm{Hz}$ can achieve raw contrasts of $10^{-4}$ and better. We confirmed that the standard deviation of intensity in difference images evolves parabolically with time at short timescales. Intensity is the square of complex amplitude, and the complex amplitude changes linearly with time for short timescales ( $\sim \mathrm{ms}$ in our case, but more generally timescales on the order of the bandwidth of the AO system). Therefore the parabolic behavior we observed is a confirmation that the intensity scales as a square of time.

The current (March 2016) SCExAO system produces images with speckles that reach brightnesses of $10^{-2}-$ $10^{-3}$ times that of the PSF core, and when the instrument is better tuned and producing $90 \%$ Strehl, the speckles will generally be kept below the $10^{-3}$ level. This is the raw contrast in a single short exposure; for reasonable integration times, the speckles move around and average to a lower level. A speckle nulling loop with a bandwidth of a few hundred $\mathrm{Hz}$ would improve this by 1-2 orders of magnitude. This, combined with coronagraphy and speckle reduction in post-processing, will enable new science opportunities such as the ability to directly image reflected-light exoplanets. An Earth-like planet around a Sun-like star has a contrast of $10^{-10}$, and this may be a feasible target in the era of thirty-meter-class telescopes once well-tuned speckle control techniques are implemented.

\section{ACKNOWLEDGMENTS}

The authors acknowledge support from NSF award AST 1106391, NASA Roses APRA award NNX 13AC14G, and the JSPS (Grant-in-Aid for Research \#23340051 and \#26220704). 


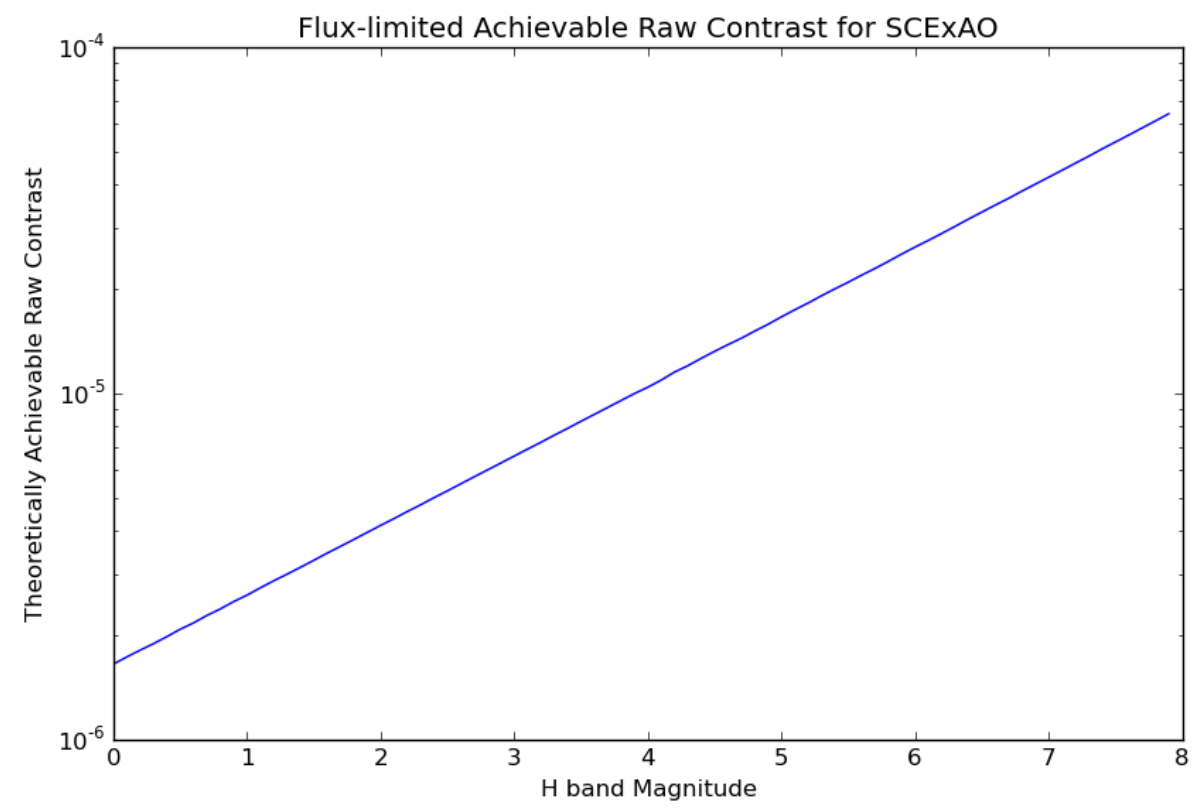

Figure 5. We calculated the raw contrast that could theoretically be achieved for a perfect SCExAO speckle nulling loop as a function of stellar magnitude. We combined Equation 1 with the equation measured in Figure 2 and solved for contrast as a function of stellar magnitude. These contrasts require a speckle nulling loop running at a speed that permits 10 photons to accumulate per speckle per integration time. We assumed an $8.2 \mathrm{~m}$ telescope, $10 \%$ system throughput, 10 photons per speckle, and $\mathrm{H}$ band observations. The overall contrast can be further improved by integrating for an extended period of time and then applying post-processing techniques.

\section{REFERENCES}

[1] Macintosh, B., Poyneer, L., Sivaramakrishnan, A., and Marois, C., "Speckle lifetimes in high-contrast adaptive optics," in [Astronomical Adaptive Optics Systems and Applications II], Tyson, R. K. and LloydHart, M., eds., Proc. SPIE 5903, 170-177 (Aug. 2005).

[2] Olivier, S., Max, C. E., Brase, J. M., Gavel, D. T., Macintosh, B., and Carrano, C., "Brown dwarfs and extrasolar planets," in [262], Rebolo, R., Martin, E., and Zapatero Osorio, M. R., eds., ASP Conference Series 134 (1998).

[3] Angel, J. R. P., "Ground-based imaging of extrasolar planets using adaptive optics," Nature 368, 203-207 (Mar. 1994).

[4] Marois, C., Lafrenière, D., Doyon, R., Macintosh, B., and Nadeau, D., "Angular Differential Imaging: A Powerful High-Contrast Imaging Technique," ApJ 641, 556-564 (Apr. 2006).

[5] Smith, W. H., "Spectral differential imaging detection of planets about nearby stars," PASP 99, 1344-1353 (Dec. 1987).

[6] Brandner, W., Apai, D., Lenzen, R., Feldt, M., and Huélamo, N., "Polarimetric Differential Imaging Studies of Stellar Environments: Disks and Planets," in [Astronomical Polarimetry: Current Status and Future Directions], Adamson, A., Aspin, C., Davis, C., and Fujiyoshi, T., eds., Astronomical Society of the Pacific Conference Series 343, 75 (Dec. 2005).

[7] Martinache, F., Guyon, O., Jovanovic, N., Clergeon, C., Singh, G., and Kudo, T., "On-sky speckle nulling with the Subaru Coronagraphic Extreme AO (SCExAO) instrument," in [Society of Photo-Optical Instrumentation Engineers (SPIE) Conference Series], Society of Photo-Optical Instrumentation Engineers (SPIE) Conference Series 9148, 21 (Aug. 2014).

[8] Martinache, F. and Guyon, O., "The Subaru Coronagraphic Extreme-AO Project," in [Society of PhotoOptical Instrumentation Engineers (SPIE) Conference Series], Society of Photo-Optical Instrumentation Engineers (SPIE) Conference Series 7440, 0 (Aug. 2009). 
[9] Guyon, O., Martinache, F., Clergeon, C., Russell, R., Groff, T., and Garrel, V., "Wavefront control with the Subaru Coronagraphic Extreme Adaptive Optics (SCExAO) system," in [Society of Photo-Optical Instrumentation Engineers (SPIE) Conference Series], Society of Photo-Optical Instrumentation Engineers (SPIE) Conference Series 8149, 8 (Oct. 2011).

[10] Minowa, Y., Hayano, Y., Oya, S., Watanabe, M., Hattori, M., Guyon, O., Egner, S., Saito, Y., Ito, M., Takami, H., Garrel, V., Colley, S., Golota, T., and Iye, M., "Performance of Subaru adaptive optics system AO188," in [Society of Photo-Optical Instrumentation Engineers (SPIE) Conference Series], Society of Photo-Optical Instrumentation Engineers (SPIE) Conference Series 7736, 3 (July 2010).

[11] Hodapp, K. W., Suzuki, R., Tamura, M., Abe, L., Suto, H., Kandori, R., Morino, J., Nishimura, T., Takami, H., Guyon, O., Jacobson, S., Stahlberger, V., Yamada, H., Shelton, R., Hashimoto, J., Tavrov, A., Nishikawa, J., Ukita, N., Izumiura, H., Hayashi, M., Nakajima, T., Yamada, T., and Usuda, T., "HiCIAO: the Subaru Telescope's new high-contrast coronographic imager for adaptive optics," in [Society of PhotoOptical Instrumentation Engineers (SPIE) Conference Series], Society of Photo-Optical Instrumentation Engineers (SPIE) Conference Series 7014, 19 (July 2008).

[12] Norris, B., Schworer, G., Tuthill, P., Jovanovic, N., Guyon, O., Stewart, P., and Martinache, F., "The VAMPIRES instrument: imaging the innermost regions of protoplanetary discs with polarimetric interferometry," MNRAS 447, 2894-2906 (Mar. 2015).

[13] Huby, E., Perrin, G., Marchis, F., Lacour, S., Kotani, T., Duchêne, G., Choquet, E., Gates, E. L., Woillez, J. M., Lai, O., Fédou, P., Collin, C., Chapron, F., Arslanyan, V., and Burns, K. J., "FIRST, a fibered aperture masking instrument. I. First on-sky test results," A $\mathscr{E} A$ 541, A55 (May 2012).

[14] Finger, G., Baker, I., Alvarez, D., Ives, D., Mehrgan, L., Meyer, M., Stegmeier, J., and Weller, H. J., "SAPHIRA detector for infrared wavefront sensing," in [Adaptive Optics Systems IV], Proc. SPIE 9148, 914817 (Aug. 2014).

[15] Leach, R. W. and Low, F. J., "CCD and IR array controllers," in [Optical and IR Telescope Instrumentation and Detectors], Iye, M. and Moorwood, A. F., eds., Society of Photo-Optical Instrumentation Engineers (SPIE) Conference Series 4008, 337-343 (Aug. 2000).

[16] Basden, A. G., Myers, R. M., and Gendron, E., "Wavefront sensing with a brightest pixel selection algorithm," MNRAS 419, 1628-1636 (Jan. 2012).

[17] Mazin, B. A., "Microwave Kinetic Inductance Detectors: The First Decade," in [American Institute of Physics Conference Series], Young, B., Cabrera, B., and Miller, A., eds., American Institute of Physics Conference Series 1185, 135-142 (Dec. 2009).

[18] Mazin, B. A., Meeker, S., Strader, M., Szypryt, P., Walter, A., Bockstiegel, C., Collura, G., Mawet, D., Jensen-Clem, R., Guyon, O., Jovanovic, N., Oppenheimer, R., and Serabyn, E., "MKIDs for Direct Imaging of Exoplanets," in [AAS/Division for Extreme Solar Systems Abstracts], AAS/Division for Extreme Solar Systems Abstracts 3, 104.07 (Dec. 2015). 